\title{
Análisis de los reportes de Proyectos de Innovación Tecnológica presentados por estudiantes del Instituto Tecnológico de Chihuahua
}

\section{Analysis of the reports of Technological Innovation Projects for students of Instituto Tecnológico de Chihuahua}

Renzo Eduardo Herrera Mendoza ${ }^{1}$

Brenda Ileana Solís Herrera ${ }^{2}$

\begin{abstract}
Resumen
La siguiente investigación muestra los resultados finales de la caracterización de los Proyectos de Innovación Tecnológica realizados por estudiantes quienes fueron asistidos por docentes del Instituto Tecnológico de Chihuahua en su participación en el Evento Nacional Estudiantil de Investigación Tecnológica en su etapa local. El objetivo es valorar los contenidos que presentan los estudiantes en los formatos al llenar la información de sus proyectos. La metodología es de enfoque cualitativo a través del análisis de los contenidos en los formatos de proyectos de acuerdo a la rúbrica del concurso. Los resultados muestran deficiencias en cuanto al modelo de negocios, enfoque hacia el mercado meta, conceptos básicos sobre lo que proponen ya que no presentan argumentos sólidos con bases científicas, tanto en su redacción, como en la presentación de sus proyectos, pues hay faltas de ortografía en la citación de la literatura
\end{abstract}

1 Renzo Eduardo Herrera Mendoza. Profesor-investigador del Centro de Investigación y Docencia de los Servicios Educativos del Estado de Chihuahua, México. Es Doctor en Administración pública y Participa en al Cuerpo Académico Política y Gestión Educativa. Correo electrónico: renzo.herrera@cid.edu.mx ID: http://orcid.org/0000-0001-6477-4476

2 Brenda Solís Herrera. Docente de la Facultad de Medicina y Ciencias Biomédicas de la Universidad Autónoma de Chihuahua, México. Es Maestra en Comunicación y forma parte del Comité Editorial de la Revista IE de la REDIECH. Correo electrónico: brenda.solish@gmail.com

ID: http://orcid.org/0000-0002-8698-8552 
consultada en donde presentan pocas fuentes y la mayoría son links de páginas de instituciones públicas. De la misma manera, falta focalizar el problema al cual van a atender. En ese sentido, también se observa que el acompañamiento del asesor, quien es docente de clase, desconoce del tema empresarial ya que no tienen negocio, o no tiene capacitación sobre lo que es un modelo de negocios, así como la parte teórica y alineación a las necesidades de vocación económica de la identidad, por lo que sus áreas de oportunidad se ven reflejadas en sus asesorías con sus estudiantes.

\title{
Palabras clave
}

Evaluación de proyectos, evaluación de estudiantes, habilidades investigativas, innovaciones tecnológicas, papel del asesor.

\begin{abstract}
The following research shows the final results of the characterization of Technological Innovation Projects carried out by students who were assisted by teachers from the Instituto Tecnológico de Chihuahua in their participation in the National Student Event of Investigation Technological in its local stage. The objective is to assess the contents that the students present in the formats when filling the information of their projects. The methodology is a qualitative approach through the analysis of the contents of the project formats according to the contest's rubric. The results show deficiencies in terms of the business model, focus on the target market, basic concepts on what they propose do not present solid arguments with scientific basis, both in their writing and in the presentation of their projects there are spelling mistakes, in the citation of the consulted literature added to that they present few sources and the majority link of pages of public institutions, it is necessary to focus the problem to which they are going to attend. In this sense, it is also observed that the accompaniment of the advisor who is a class teacher, is unaware of the business issue since they have no business or have no training on what is a business model as well as the theoretical part and alignment to the needs of economic vocation of identity, so that their areas of opportunity are reflected in their advice with their students.
\end{abstract}

\section{Keywords}

Project evaluation, student evaluation, investigative skills, technological innovations, role of the advisor. 


\section{Introducción}

En la actualidad, es común que en las instituciones académicas se hable de términos de emprendimiento e innovación, en donde incluso se planteen ferias de emprendimiento, de tal forma que algunas materias, pretenden que los estudiantes generen proyectos que terminen incubándose, para que en el corto plazo, se genere un negocio. Sin embargo, hay autores como Herrera y Álvarez (2015) quienes cuestionan ¿Qué espacios y tiempos se disponen para el aprendizaje y el desarrollo de la creatividad en las universidades?, ¿Cómo se procura el aprendizaje de la resolución de problemas desde las aulas?, ¿Se valora el desarrollo de redes de conocimiento, así como las relaciones institucionales y empresariales como actividades necesarias desde la formación de pregrado?

En los últimos años, las Instituciones de Educación Superior han buscado generar estrategias dentro de materias para proyectar desde lo académico, equipos de trabajo para impulsarlos a desarrollar algún proyecto, el cual se pretende, llegue a generar una idea de negocio. Solo que es aquí donde empiezan los problemas, ya que existen elementos en contra para que se den en realidad emprendimientos. En primer término, las mismas materias que se tienen dentro de la currícula de las carreras, no cuentan con el desarrollo de la temática adecuada, es decir, quedan demasiadas ambiguas.

Así mismo, existen limitaciones en cuanto infraestructura, tiempos en los horarios de los estudiantes, vocación de la institución para desarrollar emprendimientos; aunado a ello, los docentes, quienes tienen a cargo estas materias, no tienen negocio o no lo han tenido. Esto de entrada, evidencia la incongruencia de la institución para determinar en manos de quien enseña o da acompañamiento a estos tipos de materias, además de que no conocen de emprendimiento ni tienen cursos, ni certificaciones que les den herramientas suficientes para el correcto asesoramiento de los estudiantes.

Un estudio realizado por Alfaro, González, Parra y González (2016, p. 100) hizo ver que:

... triangular y contrastar los Syllabus en emprendimiento de las instituciones de educación superior que se ofertaban en su municipio, demostró que las competencias se orientan en todos los ciclos al desarrollo temático, al manejo de TIC, al igual que al desarrollo de habilidades, valores y actitudes necesarias para el emprendimiento y la generación de ideas de negocio y fortalecimiento del emprendimiento.

En ese sentido, es importante que las instituciones educativas "asuman transformaciones en los cuatro pilares fundamentales de su quehacer, en el 
ámbito pedagógico, epistemológico, de acción social y de gestión" (Bilbao y Vélez, 2015, p. 176). Desde esta perspectiva, la educación y la formación deben contribuir a impulsar el espíritu emprendedor con actitudes favorables, la sensibilización hacia las salidas profesionales como empresarios y las competencias (Vásquez, 2017, p. 145; Perdomo, Alcívar, Salvatierra, Martínez y Vilcacundo, 2017).

Lo anterior, es un fuerte argumento para exigir a las instituciones académicas elevar la calidad de los estudiantes que ofrecen a la sociedad y que definitivamente tiene que ir transformándose para obtener poco a poco mejores resultados. De tal suerte que las dependencias de gobierno y organismos independientes que tienen programas presupuestarios para apoyar proyectos productivos, se fortalecieran desde la academia y sobre las necesidades empresariales con vocación regional. Además es importante considerar lo que menciona Malásquez (2017) que habrá que evidenciar si ello se traduce en empresas más competitivas, en bienes y servicios de mejor calidad, en recursos humanos más especializados, en empleos dignos y salarios mejores. Si se consideran las oportunidades de acuerdo a las necesidades de estas vocaciones en sus cadenas productivas y de valor que conlleven a crear $\mathrm{y}$ fortalecer los empleos desde los egresados universitarios como posibles proveedores de servicios y productos contribuyendo al crecimiento económico (Rodríguez y Chávez, 2015, p. 36; Méndez, 2016).

Es evidente que las instituciones públicas locales aún demuestran falta de oficio para generar nivel de proyectos productivos para emprendimientos reales que lleguen a generar pymes. Hay trabajo académico por delante para las instituciones; desde dotar herramientas a los docentes tutores para fortalecerlos en materia de modelos de negocios y conocimientos conceptuales teóricos, así como la exigencia para trasmitirles a los estudiantes y que los mismos trabajen propuestas de emprendimiento, ya que estas contribuyen a apalancar el ciclo de vida de las nuevas empresas por medio de la investigación, el desarrollo y la innovación para el uso de las tecnologías de la información y finalmente que este conocimiento genere oportunidades de mercado y la producción de valor agregado (Castro, Trocel y Jiménez, 2015; Espinoza y Arredondo, 2016).

Finalmente, la escuela pública cuenta con el prestigio de formar profesionistas que el mercado local absorbe, pero su profesionalización deja mucho que desear en los servicios que ofrece el egresado, de tal forma que las empresas aún tienen que seguirlo dotando de herramientas y carácter para que termine su maduración. Y más allá del costo que conlleva esto, es la 
política pública de la SEP que permite el bajo nivel tanto académico por informar el volumen de egresados que generan los presupuestos de gobierno; sin embargo, la realidad muestra que deben ser exigidos e involucrados aún más en la vida real de las necesidades de proveeduría local, así como del propio involucramiento del sector empresarial, he ir de la mano para vivir en conjunto lo que se requiere en la vida productiva del sector económico.

\section{Metodología}

La metodología tiene un enfoque cualitativo a través del análisis de los contenidos de los formatos de proyectos, de acuerdo a la rúbrica del concurso en cuanto a su evaluación documental y presentación de proyectos. Se evaluaron ocho proyectos, los cuales estaban en las categorías de producto y servicios, los proyectos evaluados son: Cinta trasportadora QVV, Ergonomic Safe Assembly, Gas Control, Medidor Ultrasónico Producto, Secbelt, Seedgarro, Sistema para cuadripléjicos, Sound off ergonomics.

Así mismo el formato de llenado o "Memoria del Proyecto" para que los equipos redactaran la información de su proyecto tenía los siguientes apartados que se describen en la rúbrica siguiente (ENEIT, 2018):

\begin{tabular}{|c|c|c|c|c|}
\hline \multicolumn{5}{|c|}{ Descripción de la problemática identificada } \\
\hline 7 puntos & $6-4$ puntos & 3- 1 punto & 0 puntos & VALOR MÁXIMO \\
\hline $\begin{array}{l}\text { Identifica claramente la } \\
\text { problemática que resuelve } \\
\text { presentando detalladamente } \\
\text { y de forma desglosada } \\
\text { resultados de búsquedas de } \\
\text { tecnologías similares y } \\
\text { presenta una justificación } \\
\text { adecuada de la solución del } \\
\text { problema, sustentada con } \\
\text { fuentes de información } \\
\text { especializadas, destacando } \\
\text { claramente los elementos } \\
\text { diferenciadores de su } \\
\text { proyecto respaldándolo con } \\
\text { tablas comparativas. }\end{array}$ & $\begin{array}{l}\text { Identifica claramente la } \\
\text { problemática que resuelve } \\
\text { presentando detalladamente y } \\
\text { de forma desglosada } \\
\text { resultados de búsquedas de } \\
\text { tecnologías similares y } \\
\text { presenta una justificación de la } \\
\text { solución del problema con } \\
\text { referencias no confiables, } \\
\text { destacando claramente los } \\
\text { elementos diferenciadores sin } \\
\text { profundidad. }\end{array}$ & $\begin{array}{l}\text { Identifica la problemática que } \\
\text { resuelve y presenta una } \\
\text { justificación sin referencias, } \\
\text { describiendo sus elementos } \\
\text { diferenciadores sin realizar un } \\
\text { análisis. }\end{array}$ & $\begin{array}{l}\text { NO identifica la } \\
\text { problemática que } \\
\text { resuelve, NO describe de } \\
\text { manera clara su elemento } \\
\text { diferenciador y No existe } \\
\text { una justificación } \\
\text { adecuada. }\end{array}$ & 7 puntos \\
\hline \multicolumn{5}{|c|}{ Impacto del proyecto en el sector estratégico } \\
\hline 5 puntos & 4- 3 puntos & 2-1 punto & 0 puntos & VALOR MÁXIMO \\
\hline $\begin{array}{l}\text { Identifica de manera correcta } \\
\text { al sector estratégico y a la } \\
\text { industria a la que pertenece } \\
\text { dicho producto o servicio, } \\
\text { presentado referencias } \\
\text { sustentadas con datos } \\
\text { estadísticos. }\end{array}$ & $\begin{array}{l}\text { Identifica de manera correcta al } \\
\text { sector estratégico y a la } \\
\text { industria a la que pertenece } \\
\text { dicho producto o servicio. }\end{array}$ & $\begin{array}{l}\text { NO Identifica de manera } \\
\text { correcta al sector estratégico, } \\
\text { pero si identifica a la industria } \\
\text { a la que pertenece dicho } \\
\text { producto o servicio. }\end{array}$ & $\begin{array}{l}\text { NO identifica de manera } \\
\text { correcta al sector } \\
\text { estratégico al que } \\
\text { pertenece y NO identifica } \\
\text { la industria a la que } \\
\text { pertenece. }\end{array}$ & 5 puntos \\
\hline
\end{tabular}

Figura 1: Descripción de la problemática e impacto del proyecto.

Análisis de los reportes de Proyectos de Innovación Tecnológica... 
En la Figura 1, se muestra la descripción del apartado, descripción de la problemática e impacto del proyecto de acuerdo al sector, que en este caso fue producción o servicios. Cada uno contiene cinco columnas con diferente valoración para que el evaluador en base a ello pueda calificar.

\begin{tabular}{|c|c|c|c|c|}
\hline \multicolumn{5}{|c|}{ Estado de la técnica } \\
\hline 8 puntos & 7-5 puntos & 4-1 punto & 0 puntos & VALOR MÁXIMO \\
\hline $\begin{array}{l}\text { Presenta información } \\
\text { desglosada de diferentes } \\
\text { autores así como las } \\
\text { tecnologias que resuelven el } \\
\text { problema, analizando y } \\
\text { comparando los trabajos } \\
\text { consultados de fuentes } \\
\text { confiables (CONRICyT, JCR, } \\
\text { etc.), mediante cuadros } \\
\text { comparativos. Debe citar } \\
\text { todas las fuentes de } \\
\text { información consultadas de } \\
\text { acuerdo al formato de } \\
\text { referencia APA. }\end{array}$ & $\begin{array}{l}\text { Presenta información } \\
\text { desglosada de diferentes } \\
\text { autores así como las } \\
\text { tecnologías que resuelven el } \\
\text { problema, analizando y } \\
\text { comparando los trabajos } \\
\text { consultados, presenta } \\
\text { referencias bibliográficas, } \\
\text { citadas de forma incorrecta. }\end{array}$ & $\begin{array}{l}\text { Presenta información de } \\
\text { diferentes autores así como } \\
\text { las tecnologías que resuelven } \\
\text { el problema, sin analizar, } \\
\text { presenta referencias } \\
\text { bibliográficas de fuentes no } \\
\text { confiables. }\end{array}$ & $\begin{array}{l}\text { NO presenta información } \\
\text { pertinente. } \\
\text { NO presenta referencias } \\
\text { bibliográficas. }\end{array}$ & 8 puntos \\
\hline \multicolumn{5}{|c|}{ Descripción de la innovación } \\
\hline 10 puntos & 9-5 puntos & 4-1 puntos & 0 puntos & VALOR MÁXIMO \\
\hline $\begin{array}{l}\text { Los integrantes del equipo } \\
\text { son capaces de describir de } \\
\text { forma clara y precisa las } \\
\text { características y beneficios } \\
\text { de su innovación resaltando } \\
\text { sus ventajas competitivas. }\end{array}$ & $\begin{array}{l}\text { Los integrantes del equipo son } \\
\text { capaces de describir de forma } \\
\text { clara y precisa las } \\
\text { características y beneficios de } \\
\text { su innovación. }\end{array}$ & $\begin{array}{l}\text { Los integrantes del equipo son } \\
\text { capaces de describir las } \\
\text { características de su } \\
\text { innovación. }\end{array}$ & $\begin{array}{l}\text { Los integrantes del equipo } \\
\text { NO son capaces de } \\
\text { identificar y describir las } \\
\text { caracteristicas de su } \\
\text { innovación. }\end{array}$ & 10 puntos \\
\hline
\end{tabular}

Figura 2: La técnica y la descripción de la Innovación.

En la descripción de los elementos técnicos que tiene cada uno de los proyectos y de lo que se está innovando (Figura 2), se presentan los rubros que el evaluador deberá considerar para darle una ponderación de acuerdo al nivel académico, aún a pesar del semestre en el que se encuentran, por lo que la exigencia incide en la redacción y la forma de citar las fuentes consultadas, así como su habilidad para explicar por qué su producto es realmente innovador y que aún no existe. 


\begin{tabular}{|c|c|c|c|c|}
\hline \multicolumn{5}{|c|}{ Viabilidad de Mercado } \\
\hline 15 puntos & 14-8 puntos & 7-1 puntos & 0 puntos & VALOR MÁXIMO \\
\hline $\begin{array}{l}\text { Identifica claramente las } \\
\text { oportunidades e } \\
\text { impedimentos para que la } \\
\text { innovación entre al mercado, } \\
\text { así como su mercado } \\
\text { potencial y meta, justificando } \\
\text { el porqué de ellos; además } \\
\text { presentando y analizando la } \\
\text { información cuantitativa y } \\
\text { cualitativa de dichos } \\
\text { mercados. }\end{array}$ & $\begin{array}{l}\text { Identifica claramente las } \\
\text { oportunidades e impedimentos } \\
\text { para que la innovación entre al } \\
\text { mercado, así como su mercado } \\
\text { potencial y meta, justificando el } \\
\text { porqué de ellos. }\end{array}$ & $\begin{array}{l}\text { Identifica claramente las } \\
\text { oportunidades e } \\
\text { impedimentos para que la } \\
\text { innovación entre al mercado, } \\
\text { así como su mercado } \\
\text { potencial y meta. }\end{array}$ & $\begin{array}{l}\text { NO tiene claro las } \\
\text { oportunidades e } \\
\text { impedimentos para que la } \\
\text { innovación entre al } \\
\text { mercado, así como su } \\
\text { mercado potencial y } \\
\text { meta. }\end{array}$ & 15 puntos \\
\hline \multicolumn{5}{|c|}{ Pre-factibilidad técnica-económica } \\
\hline 15 puntos & 14-8 puntos & 7-1 puntos & 0 puntos & VALOR MÁXIMO \\
\hline $\begin{array}{l}\text { Identifica claramente las } \\
\text { fuentes de financiamiento } \\
\text { públicas y privadas para la } \\
\text { realización del proyecto y } \\
\text { muestra evidencia de la } \\
\text { búsqueda, entrevista o } \\
\text { contacto con instituciones u } \\
\text { organismo para la } \\
\text { generación de vínculos con } \\
\text { expertos en el área a fin, que } \\
\text { validen la pertinencia del } \\
\text { proyecto al sector } \\
\text { estratégico al que va dirigido. } \\
\text { Presenta un análisis del costo } \\
\text { del financiamiento mediante } \\
\text { tablas comparativas. }\end{array}$ & $\begin{array}{l}\text { Identifica claramente las } \\
\text { fuentes de financiamiento } \\
\text { públicas y privadas para la } \\
\text { realización del proyecto y } \\
\text { muestra evidencia de la } \\
\text { búsqueda, entrevista o } \\
\text { contacto con instituciones u } \\
\text { organismo para la generación } \\
\text { de vínculos con expertos en el } \\
\text { área a fin, sin validación del } \\
\text { proyecto. Presenta un análisis } \\
\text { del financiamiento sin } \\
\text { profundidad. }\end{array}$ & $\begin{array}{l}\text { Identifica claramente las } \\
\text { fuentes de financiamiento } \\
\text { públicas y privadas para la } \\
\text { realización del proyecto y } \\
\text { muestra evidencia de la } \\
\text { búsqueda de instituciones en } \\
\text { el área a fin, sin establecer } \\
\text { contacto. }\end{array}$ & $\begin{array}{l}\text { NO tiene claramente } \\
\text { identificadas las fuentes } \\
\text { de financiamiento } \\
\text { públicas y privadas para la } \\
\text { realización del proyecto y } \\
\text { NO muestra evidencia de } \\
\text { la búsqueda, entrevista o } \\
\text { contacto con instituciones } \\
\text { u organismo para generar } \\
\text { vínculos con expertos. }\end{array}$ & 15 puntos \\
\hline
\end{tabular}

Figura 3: Validación del Mercado y Pre-factibilidad técnica-económica.

Otro de los rubros fundamentales que potencializa a un producto o servicio para ver si pudiera ser consumible, es el enfoque al mercado y los análisis referentes a su factibilidad, tanto técnica como económica como lo muestra la Figura 4. En ese sentido, se observa que los parámetros son ambiguos y no específica claridad en la información de los elementos específicos para determinar los aspectos a evaluar. 
RECIE. Revista Electrónica Científica de Investigación Educativa Vol. 4, núm. 2, enero-diciembre 2019, pp. 1093-1105.

\begin{tabular}{|c|c|c|c|c|c|}
\hline \multicolumn{6}{|c|}{ Estrategia de propiedad intelectual } \\
\hline 10 puntos & 9-5 puntos & & 4-1 puntos & 0 puntos & VALOR MÁXIMO \\
\hline $\begin{array}{l}\text { Identifica claramente las } \\
\text { partes del proyecto } \\
\text { susceptibles a ser protegidas, } \\
\text { el tipo de figura de propiedad } \\
\text { intelectual acorde al producto } \\
\text { generado, así como las } \\
\text { estrategias para su } \\
\text { protección y evidencia del } \\
\text { proceso ante las instancias } \\
\text { adecuadas. }\end{array}$ & \multicolumn{2}{|c|}{$\begin{array}{l}\text { Identifica claramente las partes } \\
\text { del proyecto susceptibles a ser } \\
\text { protegidas, el tipo de figura de } \\
\text { propiedad intelectual acorde al } \\
\text { producto generado, así como } \\
\text { las estrategias para su } \\
\text { protección. }\end{array}$} & $\begin{array}{l}\text { Identifica claramente las } \\
\text { partes del proyecto } \\
\text { susceptibles a ser protegidas } \\
\text { y el tipo de figura de } \\
\text { propiedad intelectual acorde } \\
\text { al producto generado. }\end{array}$ & $\begin{array}{l}\text { NO define claramente las } \\
\text { partes del proyecto } \\
\text { susceptibles a ser } \\
\text { protegidas. }\end{array}$ & 10 puntos \\
\hline \multicolumn{6}{|c|}{ Habilidades para la presentación y defensa del proyecto } \\
\hline 10 puntos & 9-5 puntos & & 4-1 puntos & 0 puntos & VALOR MÁXIMO \\
\hline $\begin{array}{l}\text { La presentación debe } \\
\text { contener información } \\
\text { pertinente, realizando una } \\
\text { síntesis y presentando datos } \\
\text { y resultados de forma gráfica } \\
\text { en una secuencia lógica, } \\
\text { organizada y estructurada, } \\
\text { sin saturar las diapositivas de } \\
\text { contenidos. Los integrantes } \\
\text { del equipo manejan los } \\
\text { conceptos e información de } \\
\text { forma fluida, usando un } \\
\text { lenguaje formal y técnico } \\
\text { especializado, mostrando un } \\
\text { dominio avanzado del tema. }\end{array}$ & $\begin{array}{l}\text { La presentación debe } \\
\text { contener información } \\
\text { pertinente, realizando una } \\
\text { síntesis y presentando } \\
\text { datos y resultados de } \\
\text { forma gráfica. Los } \\
\text { integrantes del equipo } \\
\text { manejan los conceptos e } \\
\text { información de forma } \\
\text { fluida, usando un lenguaje } \\
\text { formal y técnico } \\
\text { especializado, mostrando } \\
\text { un dominio básico del } \\
\text { tema. }\end{array}$ & & $\begin{array}{l}\text { presentación debe contener } \\
\text { ormación pertinente, realizando } \\
\text { a síntesis de la información. Los } \\
\text { egrantes del equipo manejan } \\
\text { unos conceptos e información } \\
\text { forma fluida, usando un } \\
\text { guaje técnico, mostrando } \\
\text { nocimiento del tema. }\end{array}$ & $\begin{array}{l}\text { Realizan la presentación } \\
\text { con la información NO } \\
\text { organizada o } \\
\text { estructurada. Los } \\
\text { integrantes del equipo } \\
\text { muestran deficiencias en } \\
\text { el dominio del tema. }\end{array}$ & 10 puntos \\
\hline
\end{tabular}

Figura 4: Estrategia de propiedad intelectual y habilidades de defensa del proyecto.

Las estrategias para determinar que lo que plantean no tiene ya derechos de autor, de acuerdo a la propiedad intelectual, así como las habilidades para la presentación y defensa del proyecto, se explican en la Figura 4. Cabe resaltar que las reglas solo permitían que un solo estudiante expusiera. 


\begin{tabular}{|c|c|c|c|c|}
\hline \multicolumn{5}{|c|}{ Prueba del prototipo } \\
\hline 10 puntos & 9-5 puntos & 4-1 puntos & 0 puntos & $\begin{array}{l}\text { VALOR } \\
\text { MÁXIMO }\end{array}$ \\
\hline $\begin{array}{l}\text { Presenta una simulación o una } \\
\text { prueba de concepto de la } \\
\text { propuesta, que muestra de } \\
\text { manera clara la innovación } \\
\text { propuesta en su proyecto. }\end{array}$ & $\begin{array}{l}\text { Presenta un avance parcial en el } \\
\text { desarrollo de su simulación o } \\
\text { prueba de concepto de la } \\
\text { propuesta, que muestra de } \\
\text { manera clara la innovación } \\
\text { propuesta en su proyecto. }\end{array}$ & $\begin{array}{l}\text { Presenta elementos que } \\
\text { respalden el desarrollo de su } \\
\text { simulación o prueba de } \\
\text { concepto de la propuesta, que } \\
\text { muestra de manera clara la } \\
\text { innovación en su proyecto. }\end{array}$ & $\begin{array}{l}\text { Solo presenta la idea, No } \\
\text { presenta elementos que } \\
\text { respalden el desarrollo de su } \\
\text { simulación o prueba de } \\
\text { concepto, no muestra de } \\
\text { manera clara la innovación } \\
\text { propuesta en su proyecto. }\end{array}$ & 10 puntos \\
\hline \multicolumn{5}{|c|}{ Complejidad tecnológica } \\
\hline 10 puntos & 9-5 puntos & 4-1 puntos & 0 puntos & $\begin{array}{c}\text { VALOR } \\
\text { MÁXIMO }\end{array}$ \\
\hline $\begin{array}{l}\text { Presenta una innovación } \\
\text { disruptiva (un producto o } \\
\text { servicio que transforma por } \\
\text { completo una industria y } \\
\text { genera otra totalmente nueva } \\
\text { a través de una propuesta de } \\
\text { valor) empleando } \\
\text { conocimientos de frontera. }\end{array}$ & $\begin{array}{l}\text { Presenta una innovación } \\
\text { incremental (un producto o } \\
\text { servicio que crea un valor sobre } \\
\text { un producto o servicio existente } \\
\text { añadiéndole mejoras o } \\
\text { características) empleando } \\
\text { conocimientos de frontera. }\end{array}$ & $\begin{array}{l}\text { Presenta una innovación } \\
\text { incremental (un producto o } \\
\text { servicio que crea un valor sobre } \\
\text { un producto o servicio existente } \\
\text { añadiéndole mejoras o } \\
\text { características) empleando } \\
\text { conocimientos básicos. }\end{array}$ & $\begin{array}{l}\text { El proyecto No presenta } \\
\text { innovación alguna. }\end{array}$ & 10 puntos \\
\hline
\end{tabular}

Figura 5: Prueba de prototipo y complejidad tecnológica.

En cuanto a los parámetros considerados para medir la complejidad tecnológica al probar el prototipo (Figura 5), éstos se enfocan en la observación in situ para ver el desempeño y argumentos al explicar su propuesta de prototipo cuando se evalúa el diseño.

\section{Análisis de resultados}

\section{Descripción de la problemática e impacto del proyecto}

Este rubro es la parte descriptiva de explicar la problemática con elementos de fuentes de investigación primarias y de diversas índoles que debieran citar para referir la problematización de la propuesta. En ese sentido, en todos los proyectos evaluados no estaba presente un planteamiento de la problematización con cifras y datos duros así como un posicionamiento geográfico del sector al que querían impactar. En cuestión teórica y referente de literatura para sustentar la propuesta con aspectos conceptuales y citar adecuadamente en formato APA con su respectiva fuente de información al final del documento, en la mayoría no se tenía, y los que tenían no estaba adecuadamente ni citada ni la bibliografía con el formato APA.

\section{La técnica y la descripción de la Innovación}

Cuando se leían los apartados de estructuración de los aspectos técnicos, la explicación fue breve y escasa, no había referentes conceptuales con su 
respectiva cita de la fuente técnica, ni fórmulas, ni dimensiones, ni dibujos de los diseños, lo cual fue notorio en el momento de las presentaciones.

\section{Validación del Mercado y Pre-factibilidad técnica-económica}

Por lo general usan un esquema de análisis de Fortaleza, Oportunidades, Debilidades y Amenazas mejor conocido como "FODA", en donde concentraban información empírica y discrecional sin un sustento de primera fuente. Ello implica que el análisis del mercado meta y el sector a impactar, así como sus competidores, es limitado. En cuanto al estudio de factibilidad en diversos rubros fueron bastantes débiles o nulas, incluso sin idea de lo que era esta información, ya que al realizar preguntas en las cuales a los evaluadores les dejaban dudas en la información mostrada y en la exposición, las respuestas obtenidas rodeaban la pregunta o bien no tenían argumento alguno.

Estrategia de propiedad intelectual y habilidades de defensa del proyecto Se observó en todos los equipos que fue un rubro que lo tenían bien identificado en los portales de la Secretaria de Economía a través del Instituto Mexicano de Propiedad Intelectual, así como otras herramientas para localizar algún otro producto con semejantes características. De tal forma que sabían buscar e identificar cómo se investigaba este aspecto.

En el rubro de defensa del proyecto las reglas fueron que solo un estudiante realizaba la presentación, de los cuales, hubo tres equipos que sí tuvieron muy buena exposición y carácter, así como vestimenta propia para su exposición, además, al momento de responder si participaban todos los miembros del equipo, si había aspectos técnicos que no sabían, así lo hacían saber.

Por otro lado, el resto de los cinco equipos se vieron nerviosos, incluso en dos casos hubo momentos en que tenían que hacer pausa por los nervios y decían información equivoca. Sus presentaciones estaban repletas de contenido y pocas imágenes, por lo regular leían, no explicaban y veían poco a los evaluadores. Al momento de responder las dudas de los evaluadores se volteaban a ver quién respondía y dudosos de lo que iban a decir, e incluso no entendían lo que se les preguntaba, por lo que se observó que hay debilidades en las competencias de los estudiantes y del asesor para prepararlos en este rubro. 


\section{Prueba de prototipo y complejidad tecnológica}

En este apartado, se observó que hay limitantes en cuanto a recursos y apoyos para presentar sus prototipos, que a su vez está limitada además por la complejidad tecnológica, debido a que no cuentan con el tiempo suficiente. Incluso limitados en tiempos porque algunos de los estudiantes trabajaban y estudiaban, por lo que dificultaba la participación de varios de los integrantes. En cuanto a mostrar lo que realiza o hace el producto o servicio, se observó que no saben mostrar o vender su proyecto, partiendo de la necesidad que tiene el cliente y con su propuesta le darían una alternativa de solución.

\section{Conclusiones}

Esta investigación muestra la vulnerabilidad de los estudiantes así como la falta de competencias de conocimiento de modelos de negocios de los docentes que asesoran estos Proyecto de Innovación Tecnológica del Instituto Tecnológico de Chihuahua, al desarrollar estos que pretenden competir para innovar y emprender un proyecto que se esperaría iniciara con un negocio. En este sentido, al ser el objetivo el de valorar los contenidos que presentan los estudiantes en los formatos al llenar la información de sus proyectos.

Los resultados muestran deficiencias en cuanto al modelo de negocios, ya que no visualizan con precisión el enfoque hacia el mercado meta, así como los referentes de conceptos básicos sobre lo que proponen. Es decir, no presentan argumentos sólidos con bases científicas o literatura de soporte como fundamentación técnica, tanto en su redacción, como en la presentación de sus proyectos, hay faltas de ortografía. Algo sustancial para describir la propuesta, es la determinación de la problemática al cual van a atender, aspecto que no desarrollan adecuadamente con estudios diagnósticos sólidos. Aunado a ello, hay escasa o nula citación de la literatura consultada y no presentan fuentes para construir mejor sustento argumentativo, siendo la mayoría links de páginas de instituciones públicas, como por ejemplo el IMPI y EL INADEM de la Secretaria de Economía.

En lo que se refiere a la exposición de proyectos, fue evidente la limitación de los estudiantes y las habilidades de estos, por lo cual se recomienda que hay que reforzar las competencias de los estudiantes en cuanto a desarrollar este tipo de proyectos y exposición, ya que es notoria la inseguridad al exponer, con diapositivas con mucho texto y lectura, así como falta de estructura para vender un proyecto, proyección y vestimenta adecuada para la ocasión. 
RECIE. Revista Electrónica Científica de Investigación Educativa Vol. 4, núm. 2, enero-diciembre 2019, pp. 1093-1105.

Por otro lado, también se observa que el acompañamiento del asesor, quien es docente de clase, desconoce del tema empresarial ya que no tienen negocio o no tiene capacitación sobre lo que es un modelo de negocios, así como la parte teórica y alineación a las necesidades de vocación económica de la identidad, por lo que sus áreas de oportunidad se ven reflejadas en sus asesorías con sus estudiantes. En ese sentido se sugiere realizar una vinculación del sector productivo e involucramiento de empresarios y personal de las organizaciones para que den este tipo de materias, o bien los docentes inviten a talleres con platicas que les puedan dar acompañamiento durante el desarrollo de un proyecto que tenga una verdadera significancia para el sector productivo de acuerdo a las necesidades regionales.

\section{Referencias}

Alfaro, C. M. A., González, M. E. C., Parra, F. M. S., y González, D. P. (2016). Caracterización de la Formación en Emprendimiento, una Alternativa para el Crecimiento Económico del Municipio de Plato, Magdalena. Escenarios, 14(2), 86-102.

Bilbao, N. S., y Vélez, A. L. L. (2015). Las competencias de emprendimiento social, COEMS: aproximación a través de programas de formación universitaria en Iberoamérica/social entrepreneurship competences, COEMS: overview through university educational programs in latin america and spain. REVESCO: Revista de Estudios Cooperativos, (119), 159.

Castro, A. A. H., Trocel, M., y Jimenez, N. (2015). La universidad como sistema complejo y sus actores en la movilización del conocimiento a la sociedad. Novum Scientiarum, 1(1).

Espinoza, P. C., y Arredondo, S. C. (2016). La evaluación de la formación en comportamiento innovador. Alteridad, 11(1), 66-77.

Herrera, M. M., y Álvarez, J. G. C. (2015). Características para promover el emprendimiento y la innovación. Marco general para la formación en las universidades. UPIICSA. Investigación Interdisciplinaria, 1(2), 3245.

Malásquez, P. M. C. (2017). Incubadoras de empresas en México en el contexto de las relaciones universidad, empresa, gobierno. Revista de Investigación en Ciencias de la Administración, 6(11), 87-117.

Méndez, R. (2016). Formulación y evaluación de proyectos. Enfoque para emprendedores. Entornos, 29(2), 475-478.

Perdomo, P. E. Á., Alcívar, M. A. A., Salvatierra, J. X. B., Martínez, M. E. R., y Vilcacundo, J. L. G. (2017). La innovación y el emprendimiento: 
necesidades en la educación superior. Revista Didasc@lia: Didáctica y Educación. 7(4), 229-246.

Rodríguez, C. H., y Chávez, R. M. A. (2015). El desarrollo de la cultura emprendedora en estudiantes universitarios para el fortalecimiento de la visión empresarial. Ciencia Administrativa, (1), 28-37.

ENEIT (2018). Rubrica de Evaluación para la categoría PRODUCTO/SERVICIO. Evento Nacional Estudiantil de Innovación Tecnológica 2018, etapa local.

Vásquez, C. (2017). Educación para el emprendimiento en la universidad (Estudios).

\section{Agradecimientos}

Se agradece al personal del Instituto Tecnológico de Chihuahua por su consideración como evaluador de proyectos, en especial a la Ing. Itzel Palacios y al Dr. Luis Arellano organizadores del Evento Nacional Estudiantil de Investigación Tecnológica en su etapa local. 
RECIE. Revista Electrónica Científica de Investigación Educativa Vol. 4, núm. 2, enero-diciembre 2019, pp. 1093-1105. 